AN. MED INTERNA (Madrid) Vol. 18, N. $^{\circ} 5$, pp. $243-247,2001$

\title{
Recidiva de tuberculosis en los pacientes infectados por el virus de la inmunodeficiencia humana
}

\author{
F. L. LADO LADO, A. PRIETO MARTÍNEZ, A. CABARCOS ORTIZ DE BARRÓN, E. \\ CARBALLO ARCEO, E. BARRIO GÓMEZ
}

\author{
Servicio de Medicina Interna. Unidad de SIDA. Departamento de Medicina. Complejo \\ Hospitalario Universitario de Santiago. Santiago de Compostela. La Coruña
}

\author{
RELAPSE OF TUBERCULOSIS IN PATIENTS WITH HUMAN IMMU - \\ NODEFICIENCY VIRUS INFECTION
}

\begin{abstract}
RESUMEN
Objetivo: Determinar las manifestaciones clínicas de la recidiva de la tuberculosis (TB) en los pacientes infectados por el virus de la inmunodeficiencia humana (VIH) y el grado de inmunodeficiencia mostrado.

Material y métodos: Se analizan retrospectivamente 121 casos, todos ellos VIH+, diagnosticados de TB desde noviembre de 1985 hasta diciembre de 1999. Todos ellos habían cumplido correctamente el tratamiento específico de la tuberculosis.

Resultados: De los 121 casos estudiados, un total de $13(10,7 \%)$ presentaron recidiva de tuberculosis al cabo de una media de 31,8 meses (rango 1-95). En 7 casos la afectación era pulmonar (2 típica, 5 atípica), en 3 mixta (pulmonar y extrapulmonar) y en 3 extrapulmonar. La sintomatología estuvo presente en el $92 \%$ de los casos y, predominantemente, consistió en: fiebre $(92 \%)$, tos $(69 \%)$, sudoración, adelgazamiento (61\%). La hepatomegalia fue el hallazgo exploratorio más frecuente (54\%). La media de linfocitos CD4 fue de 141.7 (rango 3-406), en 8 $(61 \%)$ los recuentos era inferiores a 100 cels $/ \mathrm{mm}^{3}$. La PPD fue positiva en 1caso (14\%) y en la prueba de hipersensibilidad retardada, se observó no normoergia en todos los casos $(100 \%)$.

Conclusiones: La recidiva de la tuberculosis en pacientes infectados por el VIH se presenta con una manifiesta expresividad clínica en el ámbito de un grado severo de inmunodeficiencia. En ciertos grupos de riesgo como los usuarios de drogas por vía parenteral, debe de tenerse un alto índice de sospecha de la recidiva de la enfermedad, sobre todo en aquellos pacientes que muestren recuentos celulares bajos de linfocitos CD4.
\end{abstract}

PALABRAS CLAVE: Inmunodeficiencia. Recidiva. Tuberculosis. VIH.

\section{ABSTRACT}

Objective: To determine the clinical manifestations of the relapse of the tuberculosis $(T B)$ in the patients infected by the human immunodefi ciency virus (HIV) and the degree of immunodeficiency shown.

Material and method: They are analyzed retrospectively 121 cases, all they HIV+, diagnosed of TB from November of 1985 until December of 1999. All they fulfilled correctly the specific treatment of the tubercu losis.

Results: Of 121 studied cases, a total of $13(10.7 \%)$ presented relap se of tuberculosis finally of a mean of 31,8 months (range 1-95). In 7 cases the afectation was pulmonary (2 typical, 5 non typical), in 3 mixed (pulmonary and extrapulmonary) and in 3 extrapulmonary. The clinical sintomatology were present in $92 \%$ of the cases and, predominantly, was: fever (92\%), cough (69\%), sweating, weight loss (61\%). Hepatome galy results be the exploratory finding quite frequent $(54 \%)$. The mean of CD4+ lymphocytes was 141.7 (range 3-406), in $8(61 \%)$ the lymphocyte counts was inferior to 100 cells $/ \mathrm{mm}^{3}$. PPD was positive in 1case (14\%) and with respect to delayed-type hypersensitivity reaction test, it was observed not normoergia in all the cases (100\%).

Conclusions: Relapse of the tuberculosis in patient infected by the VIH is presented with a manifested clinical expresivity in the course of a severe degree of inmunodeficiency. In certain groups of risk as the intravenous drugs users, it must of be had a high level of suspicion of the relapse of the disease, above all in those patients that show low CD4+ lymphocyte counts.

KEY WORDS: Inmunodeficiency. Relapse. Tuberculosis. HIV.

Lado Lado FL, Prieto Martínez A, Cabarcos Ortiz de Barrón A, Carballo Arceo E, Barrio Gómez E. Recidiva de tuberculosis en los pacientes infectados por el virus de la inmunodeficiencia humana. An Med Interna (Madrid) 2001; 18: 243-247.

\section{INTRODUCCIÓN}

La tuberculosis (TB) constituye una complicación oportunista muy frecuente en el seno de la infección producida por el virus de la inmunodeficiencia humana (VIH). En estos pacientes, las manifestaciones clínicas guardan relación con la localización y extensión de la enfermedad y, a su vez, con el grado de inmunodeficiencia. Así, en los estadios iniciales, la TB se expresa de forma clásica, mientras que, en estadios avanzados, con mayor deterioro inmunológico, la TB suele presentarse con formas pulmonares atípicas, extrapulmonares y diseminadas (1-10).

En estos pacientes, las pautas para el tratamiento de la TB no difieren de las clásicas y la respuesta al régimen terapéuti- 
co completo es, en general, favorable, evidenciándose tanto una mejoría clínico-radiológica a corto plazo, como la negativización microbiológica. Cuando hay ausencia de mejoría o deterioro, debemos de pensar en la presencia de otras infecciones oportunistas, o bien que el paciente no toma correctamente la medicación $(11,12)$.

No es raro que surjan recidivas de $\mathrm{TB}$ en los pacientes infectados por el VIH. Éstas se diagnostican si, una vez concluido el tratamiento de forma adecuada y considerando al paciente curado, reaparecen, al menos, dos cultivos con un número creciente de colonias. Cuando no hay adquisición de resistencias bacterianas, ya que se presupone la realización adecuada del tratamiento, está admitido repetir el esquema terapéutico inicial $(13,14)$

El incumplimiento del tratamiento es un factor propicio en la adquisición de resistencias, y este hecho se da, con mayor frecuencia, en ciertos colectivos, principalmente en los consumidores de drogas activas $(12,15)$.

El objetivo del presente estudio es determinar las manifestaciones clínicas de la recidiva de tuberculosis en nuestro medio y su relación con el grado de inmunodeficiencia en la infección por el VIH.

\section{MATERIAL Y MÉTODOS}

Hemos realizado un estudio a partir de las historias clínicas de los pacientes infectados por el VIH y diagnosticados de TB, atendidos en nuestra Unidad de SIDA en el período que comprende desde Noviembre de 1985 hasta diciembre de 1999. No se incluyeron aquellos casos que tenían antecedentes de no haber efectuado correctamente el tratamiento específico de la tuberculosis.

La infección por el VIH se diagnosticó mediante enzimoinmunoanálisis ELISA con confirmación posterior por Western-Blot. Asimismo, se efectúo una encuesta epidemiológica para averiguar la práctica de riesgo de la infección por el VIH. En todos los pacientes, se analizó el grado de inmunodeficiencia evaluando el recuento de linfocitos CD4 y los tests cutáneos: prueba de la tuberculina (PPD) y prueba de hipersensibilidad retardada (PHR). Para el diagnóstico de la enfermedad tuberculosa en cada caso, se exigió la identificación del Mycobacterium tuberculosis (MT) por cultivo en medio de Löwestein-Jensen en alguna de las muestras obtenidas. Una vez reconocido el MT en una localización, y para estimar otras posibles localizaciones, se consideró suficiente la observación de bacilos ácido-alcohol resistentes (BAAR) por tinción de Ziehl-Neelsen en una muestra o, incluso, la simple presencia de granulomas caseosos en el estudio histológico de una porción tisular conseguida por biopsia o necropsia. También se diagnóstico la TB ante la presencia, en la radiología de tórax, de adenopatías hiliomediastínicas siempre y cuando hubiera identificación del MT en otra muestra del mismo paciente (secreción bronquial, ganglios periféricos, etc.).

Las poblaciones linfocitarias, se determinaron por citometría de flujo. Se emplearon 2 UT de tuberculina PPD RT-23 para la PPD y en la PHR se aplicó el Multitest IMC ( Instituto Meireux Lyon, Francia), que consta de siete antígenos uno de los cuales es la tuberculina y un testigo (glicerina). De este modo, se clasificaron los resultados en dos grupos: normoérgico, cuando el score resultó $10 \mathrm{~mm}$ en varones y $5 \mathrm{~mm}$ en mujeres; No normoérgico: hipoérgico, cuando el score fue entre 0-10 en hombres y 0-5 en mujeres, y anérgico, cuando el score resultó $0 \mathrm{~mm}$.

En el análisis estadístico, las variables cualitativas se expresan en frecuencia y porcentajes. Las variables cuantitativas se expresan en medias y rangos.

\section{RESULTADOS}

Se recogieron un total de 121 casos, todos ellos VIH+ y diagnosticados de TB, que habían cumplido correctamente el tratamiento. De ellos, un total de 13 casos (10\%) manifestaron recidiva de la tuberculosis, de los que 12 eran varones $(92 \%)$ con una edad media de 30,9 años. La práctica de riesgo para la infección por el VIH se relacionó, mayoritariamente (Tabla I), con el uso de drogas por vía parenteral (UDVP). En 4 casos (31\%), estaban siguiendo tratamiento antirretroviral combinado en el momento del diagnóstico de la recidiva.

\section{TABLA I}

\section{DATOS DEM O GRÁFICO S, EPIDEM IO LÓ GICO S E} INM UNOLÓGICOS

\begin{tabular}{lc}
\hline Edad media (rango) & $\mathrm{N} 0(\%)$ \\
\hline
\end{tabular}

Sexo

Varón

M ujer

\section{Prácticas de riesgo UDVP}

Otros

\section{Linfocitos CD4 \\ Media (Rango) \\ $>500$ \\ 200-500 \\ $<200$}

$141,7(3-406)$

$4(31)$

$9(69)$

$\operatorname{PPD}(+)^{*}$

\section{PHRß}

$\begin{array}{lc}\text { Normoérgicos } & 0(0) \\ \text { No Normoérgicos } & 7(100) \\ \text { Hipoérgicos } & 2(29) \\ \text { Anérgicos } & 5(71)\end{array}$

№ = Número de casos; UDVP= Usuarios de drogas por vía parenteral ß PHR = Prueba de Hipersensitibilidad Retardada. No realizada en siete casos.

* PPD $=$ Prueba de la tuberculina. No realizada en siete casos.

El tiempo medio de presentación de la recidiva, una vez concluido el tratamiento en el episodio previo, fue de 31,8 meses (rango 1-95 meses). La distribución se hizo del siguiente modo: 5 casos $(38,5 \%)$ aparecieron en el primer año; 1 $(7,7 \%)$ en el segundo año; $3(23,1 \%)$ en el tercer año; 1 $(7,7 \%)$ en el cuarto año; 0 ( $0 \%)$ en el quinto año; y los 3 casos restantes $(23,1 \%)$ entre el sexto y octavo año de haber terminado el tratamiento. 
Un total de 7 casos fueron formas pulmonares: 2 típicas (exhibían patrón radiológico clásico) y 5 atípicas (en dos casos infiltrados difusos y en tres radiología de tórax normal ); 3 casos resultaron ser formas mixtas (pulmonar y extrapulmonar): 2 pulmonar y ganglionar intratorácica, 1 pulmonar y ganglionar periférica; y 3 formas extrapulmonares: 2 ganglionares periféricas, una de ellas asociada a localización meníngea, y otra consistió en un absceso pancreático (Tabla II).

\section{TABLA II}

FORM AS DE PRESENTACIÓ N DE LA RECIDIVA DE TUBERCULOSIS EN LA INFECCIÓN POR VIH

\begin{tabular}{lcc}
\hline Tuberculosis & $\mathrm{N}$ & $\%$ \\
\hline Pulmonar & 7 & 54 \\
Extrapulmonar & 3 & 23 \\
M ixta & 3 & 23 \\
Total & 13 & 100 \\
\hline
\end{tabular}

La clínica resultó poco expresiva en un 1 caso (8\%), incluso, asintomática. En los 12 casos restantes (92\%) la sintomatología predominante fue: fiebre $(92 \%)$; tos $(69 \%)$, en un único caso, hemoptoica; sudoración y adelgazamiento $(61 \%)$. Los hallazgos exploratorios más usuales comprendieron: hepatomegalia (54\%), esplenomegalia (38\%) y adenomegalias (31\%). Se objetivó patología asociada en dos casos (15\%): uno de ellos neumonía por Pneumocystis carinii y otro candidiasis esofágica. En un paciente se constató resistencia a isoniazida en el episodio de recidiva y falleció en el curso de una toxoplasmosis cerebral.

El diagnóstico se fundamentó en la mayoría de los casos, en el examen de muestras respiratorias, y de ganglio, por el aislamiento de MT en cultivo de Löwestein-Jensen, cuyo fro- tis en esputo espontáneo o inducido resultó positivo en tres casos $(30 \%)$ y en dos casos $(67 \%)$ en el lavado broncoalveolar. En ganglios periféricos las localizaciones fueron cervicales, supraclaviculares y axilares en la mitad de los casos, e inguinales en uno. La histología fue característica, en todos los casos, con confirmación microbiológica en el 75\%.

La media de linfocitos CD4 fue de 141,7 células $/ \mathrm{mm}^{3}$ (rango 3-406) mientras que en el episodio inicial la media fue de 291,9 (rango 17-693) (Tabla III). Al estratificar dicho parámetro en tres niveles (Tabla I): >500, 200-500 y <200 la distribución fue la siguiente: $0(0 \%), 4(31 \%)$ y $9(69 \%)$ respectivamente, de éstos últimos, un total de 8 (89\%) tuvieron recuentos inferiores a 100 células $/ \mathrm{mm}^{3}$.

En el episodio de recidiva los tests cutáneos se realizaron en un total de 7 casos ya que los restantes habían mostrado anergia en controles previos; la PPD fue positiva en 1 caso (14\%) y con respecto a la PHR, se observó no normoergia en todos ellos (100\%) de los que $2(29 \%)$ eran hipoérgicos (Tabla I). Por el contrario, en el episodio inicial, la PPD fue positiva en 4 casos (31\%) y se observó no normoergia en 12 casos $(92 \%)$ de los que 5 (42\%) eran hipoérgicos (Tabla III).

\section{DISCUSIÓN}

Los pacientes $\mathrm{TB} \mathrm{VIH}+$ tienen un bajo riesgo de recidiva durante el primer año después de completado el tratamiento estimada en un $5 \%$ (11). En este estudio, encontramos que, algo más de un tercio de las recidivas evaluadas acontecieron durante el primer año tras haber finalizado el tratamiento específico, por lo cual podemos resaltar que, dicho evento es más usual en el periodo de tiempo mencionado.

En cuanto a las formas de presentación de la recidiva, los resultados obtenidos están en consonancia con lo comentado en la literatura (5,11,16-19), siendo la afectación pulmonar mayoritaria y destacando, también, una alta incidencia de for-

TABLA III

RESULTADOS INM UNO LÓ GICOS (LINFO CITOS CD 4 Y TESTS CUTÁNEO S) O BTENIDOS EN EL EPISO DIO INICIAL Y RECIDIVA DE LA TUBERCULOSIS

\begin{tabular}{|c|c|c|c|c|c|c|}
\hline Paciente & CD4 i & CD4 r & PPD i & PPD $r$ & PHR i & PHR r \\
\hline 1 & 200 & 113 & Negativo & Negativo & Anergia & Anergia \\
\hline 2 & 693 & 323 & Negativo & Positivo & Anergia & Hipoergia \\
\hline 3 & 142 & 17 & Negativo & Negativo & Hipoergia & Anergia \\
\hline 4 & 17 & 3 & Negativo & Negativo & Anergia & Anergia \\
\hline 5 & 182 & 89 & Positivo & Negativo & Hipoergia & Hipoergia \\
\hline 6 & 481 & 40 & Positivo & Negativo & Anergia & Anergia \\
\hline 7 & 56 & 260 & Negativo & N R & Hipoergia & NR \\
\hline 8 & 266 & 392 & Negativo & Negativo & Anergia & Anergia \\
\hline 9 & 371 & 80 & Negativo & N R & Hipoergia & NR \\
\hline 10 & 220 & 10 & Positivo & $\mathrm{NR}$ & Hipoergia & $\mathrm{NR}$ \\
\hline 11 & 137 & 30 & Negativo & $\mathrm{NR}$ & Anergia & $\mathrm{NR}$ \\
\hline 12 & 417 & 80 & Positivo & N R & Ergia & $\mathrm{NR}$ \\
\hline 13 & 613 & 406 & Negativo & $\mathrm{NR}$ & Anergia & $\mathrm{NR}$ \\
\hline
\end{tabular}

\section{N R.- No realizado}

CD4 i.- Linfocitos CD4 en el episodio inicial de tuberculosis; CD4.- Linfocitos CD4 en la recidiva de tuberculosis.

PPD i.- Prueba de la tuberculina en el episodio inicial de tuberculosis; PPD r.- Prueba de la tuberculina en la recidiva de tuberculosis.

PHR i.- Prueba de hipersensibilidad retardada en el episodio inicial de tuberculosis; PHR r.- Prueba de hipersensibilidad retardada en la recidiva de tuberculosis 
mas extrapulmonares (ya sean aisladas o en el contexto de formas diseminadas incluyendo también participación pulmonar asociada). Dentro de las localizaciones extrapulmonares, la ganglionar es, habitualmente, la más común (16-18). Sin embargo, en contraste con lo descrito por otros autores (20), que relatan una elevada frecuencia de tuberculosis extrapulmonar en la recidiva de TB, en nuestra serie representa en torno al 50\%, hallándose asociada a TB pulmonar en la mitad de los casos.

Con respecto a las manifestaciones clínicas, éstas estuvieron presentes en el momento del diagnóstico, en casi la totalidad de los casos analizados. Los síntomas son similares a los referidos, en general, en otras series (17-19,21-26), con predominio de fiebre y de síntomas generales o respiratorios, relacionados con la afectación pulmonar y/o extrapulmonar de la TB.

Entre los hallazgos exploratorios, el más representativo es la presencia de hepatomegalia seguido de la esplenomegalia y adenomegalias en alguna localización. En este sentido, tal y como hemos comentado en otros estudios (18), no hemos podido demostrar, de forma sistemática, la localización de la enfermedad por biopsia hepática, y aunque la hepatomegalia pueda deberse a otras muchas causas en la infección por VIH (27) es muy probable que tuviera analogía con la TB. Otro tanto podríamos decir del frecuente hallazgo de esplenomegalia.

Con respecto al grado de inmunodeficiencia, valorado por el recuento de linfocitos CD4, es importante subrayar que los recuentos fueron inferiores a 100 células $/ \mathrm{mm}^{3}$ en más de la mitad de los casos, y es llamativo el grado de anergia cutánea encontrado en los tests cutáneos Ello, en nuestra experiencia (9) nos hace suponer que la recidiva de la TB en estos pacientes surge en el contexto de un grado severo de inmunodeficiencia.

Como es sabido, la respuesta de los pacientes que completan el régimen terapéutico es, ordinariamente, favorable. Cuando hay ausencia de mejoría o deterioro debemos pensar, entre otras circunstancias, en el incumplimiento del tratamiento que aparece estimado con mayor frecuencia en ciertos colectivos como UDVP, vagabundos, alcohólicos y en pacientes infectados por el VIH, lo cual puede conllevar un aumento de resistencias $(12,15)$. En este sentido, recalcamos que, en nuestro estudio, la mayoría de los casos eran UDVP.

Una alternativa, en estos casos es el tratamiento supervisado en el que se administran pautas terapéuticas bajo observación directa, con el fin de garantizar el buen cumplimiento del mismo. Varios estudios $(28,29)$ realizados en estas condiciones, han demostrado que un régimen terapéutico prolongado con respecto a uno corto reduce la tasa de recidiva pero no la de supervivencia ante recuentos celulares de linfocitos CD4 muy bajos (inferior a 200 células $/ \mathrm{mm}^{3}$ ). Sin embargo, los recuentos inferiores a 100 células $/ \mathrm{mm}^{3}$ pueden influir en la aparición de recidivas (20), hecho que ha sucedido en más de la mitas de nuestros casos.

Por último, cabe destacar, que sólo un tercio de los casos registrados en nuestro estudio cumplían tratamiento antirretroviral. En este sentido y al igual que otros autores (30), pensamos que con el advenimiento de nuevas alternativas terapéuticas en los pacientes infectados por el VIH, el pronóstico ha cambiado sustancialmente, siendo más favorable; ello hace necesario ampliar los estudios para asentar el riesgo de recidiva en más de 1 ó 2 años después de completar el tratamiento antituberculoso.

Como resumen de lo expuesto, la recidiva de la tuberculosis en pacientes infectados por el VIH, se presenta con una manifiesta expresividad clínica, en el ámbito de un grado severo de inmunodeficiencia. A pesar de su baja frecuencia, en ciertos grupos de riesgo como los UDVP, debe de tenerse un alto índice de sospecha de la recidiva de la enfermedad, sobre todo en aquellos pacientes que mostrasen recuentos celulares bajos de linfocitos CD4. Para finalizar, resulta imprescindible que el diagnóstico de esta enfermedad, en estas situaciones, no pase desapercibido para evitar la extensión en el propio enfermo y contribuir al control eficaz de la TB.

\section{Bibliografía}

1. Pitchenik AE, Cole C, Rusell BW, Fischl MA, Spira TJ, Snider DE. Tuberculosis, atypical mycobacteriosis and the acquired immunodeficiency syndrome among Haitian and non-Haitians patients in South Florida. Ann Intern Med 1984; 101: 641-645.

2. Sunderam G, McDonald RJ, Maniatis T, Oleske J, Kapila R, Reichman. Tuberculosis as a manifestation of the acquired immunodeficiency syndrome (AIDS). JAMA 1986; 256: 362-366.

3. Theuer CP, Hopewell PC, Elias D,Schecter GF, Rutherford GW, Chaisson RE. Human immunodeficiency virus infection in tuberculosis patients. J Infect Dis 1990; 162: 8-12

4. Shafer RW, Chirgwin KD, Glatt AE, Dahdouh A, Landesman SH, Suster B. HIV prevalence, immunosuppresion, and drug resistance in patients with tuberculosis in an area endemic for AIDS. AIDS 1991; 5: 399-405.

5. Jones BE, Young SMM, Antoniskis D, Davidson PT, Kramer F, Barnes PF. Relationship of the manifestations of tuberculosis to CD4 cell counts in patients with human immunodeficiency virus infection. Am Rev Respir Dis 1993; 148: 1292-1297.

6. Keiper MD, Beumont M, Elshami A, Langlotz CP, Miller WT. CD4 T Lymphocyte count and the radiographic presentation of pulmonary tuberculosis. A study of the relationship between these factors in patients with human immunodeficiency virus infection. Chest 1995; 107: 74-80.

7. Martínez Vázquez JM, Cabarcos Ortíz de Barrón A, Barrio Gómez E. Tuberculosis e infección por VIH: de la clínica a la prevención. An Med Interna (Madrid) 1997: 14: 363-368.

8. Perlman DC, El-Sadr WM, Nelson ET, Matts JP, Telzak EE, Salomon $\mathrm{N}$, et al. Variation of chest radiographic patterns in pulmonary tuberculosis by degree human immunodeficiency virus-related immunosuppression. Clin Infect Dis 1997; 25: 242-246.

9. Lado Lado FL, Barrio Gómez E, Carballo Arceo E, Cabarcos Ortíz de Barrón A. Clinical presentation of tuberculosis and the degree of immunodeficiency in patients with HIV infection. Scand J Infect Dis 1999; 31: 387-391.

10. Lado Lado FL, Barrio Gómez E, Carballo Arceo E, Cabarcos Ortíz de Barrón A. Pulmonary tuberculosis with normal radiographs in HIVimmunodeficient patients. AIDS 1999; 13: 1146-1147.

11. Small PM, Schecter GF, Goodman PC, Sande MA, Chaisson RE, Hopewell PC. Treatment of tuberculosis in patients with advanced human immunodeficiency virus infection. N Engl J Med 1991; 324: 289-294. 
12. Ackah AN, Coulibaly D, Digbeu H, Diallo K, Vetter KM, Coulibaly I$\mathrm{M}$, et al. Response to treatment, mortality, and CD4 lymphocyte counts in HIV-infected persons with tuberculosis in Abidjan, Côte d'lvoire. Lancet 1995; 345: 607-610.

13. Consenso nacional para el control de la tuberculosis en España. Grupo de trabajo sobre tuberculosis. Med Clin (Barc) 1992; 98: 24-31.

14. Recomendaciones del Consejo Asesor Clinico del Plan Nacional sobre el SIDA. Tuberculosis e infección por VIH. Edita: Ministerio de Sanidad y Consumo 1995; 2 : 1-12.

15. Pablos-Méndez A, Knirsch ChA, Barr RG, Lerner BH, Frieden ThR Nonadherence in tuberculosis treatment: predictors and consequences in New York city. Am J Med 1997; 102: 164-170.

16. Shafer RW, Kim DS, Weiss JP, Quale JM. Extrapulmonary tuberculosis in patients with human immunodeficiency virus infection. Medicine (Baltimore) 1991; 70: 384-397.

17. Laguna F, Adrados M, Díaz F, Martínez R, García Aguado C, Puente S et al. AIDS and tuberculosis in Spain. A report of 140 cases. J Infect 1991; 23: 139-144.

18. Lado Lado FL, Barrio Gómez E, Cabarcos Ortíz de Barrón A, Carballo Arceo E, Sánchez Leira J, Pérez del Molino ML, Antúnez López JR. Tuberculosis e infección por el virus de la inmunodeficiencia humana. Presentación clínica y diagnóstico. An Med Interna (Madrid) 1998; 15 : 415-420.

19. Lado Lado FL, Barrio Gómez E, Carballo Arceo E, Cabarcos Ortíz de Barrón A. Tuberculosis e infección por el virus de la inmunodeficiencia humana: manifestaciones clínicas y rendimiento de procedimientos diagnósticos según las distintas formas de localización de la enfermedad. An Med Interna (Madrid) 2000; 17: 13-18.

20. Pulido F, Peña JM, Rubio R, Moreno S, González J, Guijarro C, et al. Relapse of tuberculosis after treatment in human immunodeficiency virus-infected patients. Arch Intern Med 1997; 157: 227-232.

21. Modilevsky T, Sattler FR, Barnes PF. Mycobacterial disease in patients with human immunodeficiency virus infection. Arch Intern Med 1989; 149: 2201-2205.

22. Nambuya A, Sewankambo N, Mugerwa J, Goodgame R, Lucas S. Tuberculosis lymphadenitis associated with human immunodeficiency virus (HIV) in Uganda. J Clin Pathol 1988; 41: 93-96.

23. Kramer F, Modilevsky T, Waliany AR, Eedom JM, Barnes PF. Delayed diagnosis of tuberculosis in patients with human immunodeficiency virus Infection. Am J Med 1990; 89: 451-456.

24. Shriner KA, Mathisen GE, Goetz MB. Comparison of Mycobacterial limphadenitis among persons infected with human immunodeficiency virus and seronegative controls. Clin Infect Dis 1992; 15: 601-605.

25. Pastores SM, Naidich DP, Aranda CP, McGuinnes G, Rom WN. Intratoracic adenopathy associated with pulmonary tuberculosis in patients with human immunodeficiency virus infection. Chest 1993; 103: 14331437.

26. Fernández Revuelta A, Arazo Garces P, Aguirre Errasti JM, Arribas Llorente JL. Tuberculosis pulmonar: diferencias entre pacientes seropositivos y seronegativos para el virus de la inmunodeficiencia adquirida. An Med Interna (Madrid) 1993; 10: 381-385.

27. Chaisson RE, Slutkin G. Tuberculosis and human immunodeficiency virus infection. J Infect Dis 1989; 159: 96-100.

28. Perriëns JH, St. Louis ME, Mukadi YB, Brown C, Prignot J, Pouthier F, et al. Pulmonary tuberculosis in HIV-infected patients in Zaire. A contolled trial of treatment for either 6 or 12 months. N Engl J Med 1995; 332: 779-784.

29. Chaisson RE, Clermont HC, Holt EA, Cantave M, Johnson MP, Atkinson J, et al. Six-month supervised intermittent tuberculosis therapy in Haitian patients with and without HIV infection. Am J Respir Crit Care Med 1996; 154: 1034-1038.

30. Shafer RW, Edlin BR. Tuberculosis in patients infected with human immunodeficiency virus: perspective on the past decade. Clin Infect Dis 1996; 22: 683-704. 